\title{
Factors Affecting the Compliance of Curative Intent Treatment in Patients with Head and Neck Cancer
}

\author{
Ting-Chieh Huang ${ }^{1}$, Po-Yueh Chen ${ }^{1}$, Luong Huu Dang ${ }^{2}$, and Shih Han Hung ${ }^{1}$ \\ ${ }^{1}$ Taipei Medical University \\ ${ }^{2}$ Ho Chi Minh City University of Medicine and Pharmacy
}

June 28, 2021

\begin{abstract}
Abstract Objective: This study aims to investigate the factors that lead to poor compliance in initiating the treatment in patients with newly diagnosed head and neck cancers. Design: Retrospective cohort study Particpants: Data from a total of 271 patients were retrieved and analyzed. After excluding patients with (1) multiple cancers (2) inconclusive diagnoses, (3) rare primary cancer sites, such as the nasal cavity, paranasal sinuses, or salivary glands and (4) primary site unknown, a total of 194 patients were included in this study. Main outcome measures: The variables included in the analysis were age, gender, primary cancer site, $\mathrm{T}$ stage, $\mathrm{N}$ stage, M stage, overall stage (I-IV), patient's residential distance, and the impact of COVID-19. Univariate and multivariate analyses were employed to evaluate the significance of these variables in regard to affecting the time to receiving on-time treatment based on the specialist's suggestion. Results: Upon multivariate analysis, the primary site, stage, and residential distance were significantly associated with initial compliance $(\mathrm{P}<0.05)$. The impact of COVID-19 showing a borderline significance $(\mathrm{p}=0.137)$. Conclusion: The primary site, overall stage, patient living distance to the healthcare facility, and the impact of COVID-19 might affect the compliance of initiating a curative-intent treatment in patients with newly diagnosed head and neck cancers.
\end{abstract}

Factors Affecting the Compliance of Curative Intent Treatment in Patients with Head and Neck Cancer

\section{Abstract}

Objective: This study aims to investigate the factors that lead to poor compliance in initiating the treatment in patients with newly diagnosed head and neck cancers.

Design: Retrospective cohort study

Particpants: Data from a total of 271 patients were retrieved and analyzed. After excluding patients with (1) multiple cancers (2) inconclusive diagnoses, (3) rare primary cancer sites, such as the nasal cavity, paranasal sinuses, or salivary glands and (4) primary site unknown, a total of 194 patients were included in this study.

Main outcome measures: The variables included in the analysis were age, gender, primary cancer site, T stage, $\mathrm{N}$ stage, M stage, overall stage (I-IV), patient's residential distance, and the impact of COVID-19. Univariate and multivariate analyses were employed to evaluate the significance of these variables in regard to affecting the time to receiving on-time treatment based on the specialist's suggestion.

Results: Upon multivariate analysis, the primary site, stage, and residential distance were significantly associated with initial compliance $(\mathrm{P}<0.05)$. The impact of COVID-19 showing a borderline significance $(\mathrm{p}=0.137)$. 
Conclusion: The primary site, overall stage, patient living distance to the healthcare facility, and the impact of COVID-19 might affect the compliance of initiating a curative-intent treatment in patients with newly diagnosed head and neck cancers.

\section{Keypoint}

1. Patient's poor coordination for cancer treatment may cause unsatisfactory outcomes.

2. The primary site, overall stage, patient living distance significantly affect the compliance of initiating the treatment.

3. Patients' actual residence areas and their transportation methods, even their caregiver's transportation methods, should be investigated initially.

4. Any possible aids or subsidies should be considered if a transportation barrier is noticed during the initial interaction with the patients.

5. A trend of increased initial treatment compliance after the COVID-19 pandemic is observed. The hardware and software resources should be re-examined and reinforced if the current resources are incapable of managing the expected increase in the volume of these patients after the COVID-19 pandemic.

\section{Introduction}

Head and neck cancer is globally increasing owing to rising rates of tobacco use and human papillomavirus infection and is among the most common cancers worldwide, with high prevalence rates in South Asia, Brazil, and central Europe.[1,2] With the development of multimodal treatments, including immunotherapy, the locoregional control rate and the overall survival of advanced head and neck cancer patients has improved significantly in recent decades.[3] However, the outcome may still be unsatisfactory because of poor coordination with the patient.[4]

It is already well accepted that interruptions in radiotherapy are associated with decreased survival in headand-neck cancer patients. $[5,6]$ Additionally, a delayed time of 60 days to the initiation of treatment is associated with decreased overall survival and increased recurrence.[7] In general, the treatment outcome is influenced by the initiation of treatment, postoperative radiotherapy, and the time from surgery through the completion of postoperative radiotherapy, also called the treatment package time (TPT).[8-10] Once the treatment plan has started, countermeasures to factors already known to result in poor treatment compliance can be adopted more quickly as the patients in the treatment process are already under specific surveillance. What might be even more challenging is identifying factors that result in patient hesitation, even regarding the initiation of treatment once the diagnosis is made. Unfortunately, few studies have contributed to understanding these initial non-compliance factors.

The purpose of this study is to retrospectively review and analyze factors that lead to non-compliance with treatment initiation by patients with newly diagnosed head and neck cancers.

\section{Materials and methods}

\subsection{Study population}

Data from patients diagnosed with head and neck cancer (oral cavity, oropharynx, laryngopharynx, and nasopharynx) between January 1, 2018, and September 30, 2020, at <blinded for review > Hospital were retrospectively collected and analyzed. Data from a total of 271 patients were retrieved from our database. After excluding patients with (1) multiple cancers (2) inconclusive diagnoses, (3) rare primary cancer sites, such as the nasal cavity, paranasal sinuses, and salivary glands and (4) primary site unknown, a total of 194 patients were included in this study. The age distribution ranged from 27 to 93 years, with an average age of 58.0 years, and the male/female ratio was 4.87:1. Patients were divided into two groups, namely, the delayed group with 76 patients who did not receive standard treatment under medical advice after cancer diagnosis within a month and the on-time group with 118 cases who received standard treatment under medical advice after cancer diagnosis within a month. 


\subsection{Study design}

Patients enrolled in this study were divided into two groups. The on-time group was defined as patients receiving treatment following their doctor's schedule after diagnosis and a cancer staging examination in $<$ blinded for review $>$ hospital. The delayed group was defined under the following situations: (1) the patient did not follow the doctor's treatment plan after diagnosis and/or cancer staging in <blinded for review $>$ hospital or (2) the patient did not follow the doctor's treatment recommendations after diagnosis and/or cancer staging in another hospital and came to $<$ blinded for review $>$ hospital without any referral for further consultation and management.

The factors which may influence the compliance were categorized by age, gender, primary cancer site (the oral cavity, nasopharynx, oropharynx, and hypopharynx-larynx), T stage, $\mathrm{N}$ stage, $\mathrm{M}$ stage, overall stage (I-IV), the patient living inside or outside the territorial dominion of $<$ blinded for review $>$ hospital (Figure 1; the Wenshan district of Taipei city, and the Shenkeng district, Pinglin district, and Shiding district of New Taipei city), and before or after the impact of COVID-19, as defined by the patient visiting our hospital before or after March 1, 2020, at which time the Taiwanese government started to raise intensive border and social activity restrictions.

\subsection{Multidisciplinary team care}

The treatment recommendations and planning of each patient were conducted by the head and neck tumor board team of specialists including otolaryngologists, oral and maxillofacial surgeons, medical oncologists, radio-oncologists, pathologists, radiologists, radiology nuclear medicine physicians, rehabilitation physicians, nutritionists, social workers and case managers.

\subsection{Ethical approval}

All procedures performed in studies involving human participants followed the ethical standards approved by the <blinded for review>-JIRB. (Approval Number: N202101050)

2.5 Statistical methods

Data were analyzed using SPSS version 16.0 (IBM, Armonk, New York, USA). Group comparisons of normally distributed quantitative parameters were analyzed using the t-test and categorical parameters with an $\chi^{2}$ test to examine the difference between patient compliance and the patient-related factors of age, gender, primary cancer site, $\mathrm{T}$ stage, $\mathrm{N}$ stage, $\mathrm{M}$ stage, overall stage, residency related to the territorial dominion of $<$ blinded for review $>$ hospital, and the impact of COVID-19.

Binary logistic regression analysis was further performed to explore the association between patient compliance and potential factors. Independent variables that were included in the binary logistic regression model were chosen if the p-value was less than 0.3 , as determined by a chi-square test/t-test. Significance was defined as a p-value of less than 0.05 .

\section{Results}

\subsection{Patient Demographics and Descriptive Statistics}

Between January 2018 and September 2020, a total of 194 patients fulfilled the inclusion criteria and were included in the study. Of these patients, 118 of them were placed in the on-time group, and 76 of them were placed in the delayed group. The age distribution ranged from 27 to 93 years, with an average age of $58.3 \pm 12.1$ years, and the male/female ratio was 4.87:1.

\subsection{Factors affecting the Compliance with Curative Intent Treatment}

Table 1 shows the factors that potentially influence the initial compliance with the standard cancer treatment recommendations. There was no significant difference in patient compliance by age or gender. The patients who had oral cavity cancers and nasopharyngeal cancers were more likely to delay their treatment $(50.0 \%$ vs. $48.3 \%$ and $30.3 \%$ vs. $15.3 \%$, respectively, intra-group differences $\mathrm{p}=0.021$ ). The non-compliance rate was 
gradually higher with the increasing stage of cancer, with $56.9 \%$ of patients at stage IV having treatment delays (intra-group differences $\mathrm{p}=0.007$ ). Patients living far from the hospital tended to delay treatment compared to those who lived in the < blinded for review $>$ territorial dominion $(56.9 \%$ vs. $34.7 \%$, respectively, $\mathrm{p}=0.003)$. The compliance percentage was higher after the influence of the COVID pandemic $(30.5 \%$ vs. $15.8 \%, \mathrm{p}=0.026)$.

Table 2 shows a multivariate analysis of factors affecting patient compliance with treatment. In the binary logistic regression analysis, the risk of non-compliance was borderline different between the oral cavity vs. oropharynx ( $\mathrm{p}=0.092 ; 95 \%$ CI: $0.083-1.207)$ and significantly between the nasopharynx vs. oropharynx $(\mathrm{p}=$ 0.045; 95\% CI: 0.049-0.960). The patients who had stage IV cancers showed a higher rate of delayed treatment than the first stage $(\mathrm{p}=0.004 ; 95 \%$ CI: 0.793-0.585). Patients living far from the hospital were more likely to delay treatment than those living in the $<$ blinded for review $>$ territorial dominion $(\mathrm{p}=0.013 ; 95 \%$ CI: 0.191-0.820). A trend of increased initial treatment compliance after the COVID-19 pandemic can still be observed with a p-value of 0.137 (95\% CI: 0.803-4.942).

\section{Discussion}

In this study, we found that factors including primary site, stage, distance to the hospital, and even the influence of the COVID-19 pandemic might affect the compliance of curative-intent treatment in patients with newly diagnosed head and neck cancers. The findings in this study provide important information about the factors influencing the intention and initiation of the recommended treatments, which is also very important but seldom addressed in the existing literature compared to issues regarding treatment compliance during head and neck cancer therapy.

The importance of treatment compliance has been broadly addressed in almost every human disease; the ideal outcome is never to be expected for a particular treatment if the treatment compliance is compromised.[11] Many studies have already addressed the importance of compliance during treatment in the field of head and neck cancers. Patel et al. conducted a study to determine if poor compliance with chemoradiation results in an increased rate of persistent neck disease. Their study included 40 patients with N+ stages III/IV squamous cell carcinoma of the upper aerodigestive tract treated with curative-intent chemoradiation who underwent subsequently planned neck dissection. The authors demonstrated that noncompliance was the only variable that had a significant correlation with positive pathology results.[12]

Nevertheless, what if the patient has no intention to engage in treatment or is unwilling to accept the standard treatment recommendations? While it is generally agreed that timely care regarding the initiation of treatment, postoperative radiotherapy, and treatment package time is associated with survival for patients with head and neck cancer, there are still many issues left unanswered, including identifying barriers to timely care, which we would like to investigate in this study to hopefully help generate strategies to overcome these problems. [9] Liao et al. reported that patients with a delay in time to treatment initiation exceeding 60 days had poorer survival and a greater risk of recurrence.[7] More recently, Chang, Y. L., et al. examined 1095 HNC patients to identify the factors and reasons impacting the discordance with the treatment plan in head and neck cancer patients and to compare the differences between the concordant group and the discordant group. The authors found that patients with advanced cancer stages, advanced age, and treatment plans of best supportive care (BSC) or surgery combined with radiation (RT), chemotherapy (CT), or chemoradiation (CCRT) were more likely to have discordance with their treatment plan. [13]

In 2006, under the United Kingdom NHS system, Duvvi et al. proposed a two-week rule for suspected head and neck cancer and reported that compliance with the two-week referral guidelines was associated with a higher cancer detection rate and hopefully demonstrated an improvement in outcome.[14] In India, it has been demonstrated that early treatment decisions and referral could significantly improve patient dropout rates and possibly compliance with treatment.[15] The authors proposed that the decentralization of cancer care is urgently needed to manage the high numbers of patients presenting to tertiary care centers. Many of the problems revealed by these studies seem to be highly related to healthcare availability issues. This finding makes our study result even more interesting, as in Taiwan, over $98 \%$ of the population is covered 
by the national health insurance [16, 17]; with such a high level of healthcare availability, affordability, and accessibility, what is left to substantively influence patients' initial treatment intentions might be especially noteworthy. We believe that the factors revealed in our study are less likely to be resolved along with socio-economic growth, which often leads to an increase in health investments. [18, 19]

In our study, patients with stage IV diseases tend to have a high chance of not being compliant with our treatment recommendations. While a patient with higher severity is usually more likely to seek more treatment options in dealing with higher threats to their health, it is interesting that the primary site also influences the initial treatment intentions. The result might be associated with the impact of oral cavity cancer treatment, which remains a major problem as treatments in the oral cavity very often lead to a significant alteration in appearance, speech, smell, and swallowing functions.[20, 21] Interestingly, our study also demonstrated that patients with nasopharyngeal cancer tend to be non-compliant. Compared to oral cavity cancer patients, these patients belong to a completely different entity, as patients with nasopharyngeal cancer tend to have indolent symptoms and signs; they might subjectively feel they are not critically ill, which is a factor that encourages them to spend more time searching for second opinions.[22]

The finding that distance significantly influenced the initial treatment intentions is particularly important in this study, especially as demonstrated in Taiwan. With a crowded population under a very high density of available healthcare providers, it is noteworthy that a district classified as being outside the $<$ blinded for review $>$ Hospital territorial dominion is actually very often still within a one-hour driving distance to the hospital. It has already been demonstrated in patients with colorectal cancer that patients with uncontrolled pain or less social support have greater odds of transportation barriers and the authors concluded that inquiring about accessible transportation should become a routine part of cancer care.[23] More recently, a study investigating the role of transportation barriers in cancer patients' decision-making regarding the treatment process demonstrated that lack of access to transportation has a significant impact on cancer patients' decisions concerning stopping or continuing treatments.[24] This study also showed that limited access to private vehicles will likely lead to the stopping of radiotherapy. Based on these findings, we recommend that patients' actual residence areas and their transportation methods, even their caregiver's transportation methods, should be investigated initially. Furthermore, any possible aids or subsidies should be considered if a transportation barrier is noticed during the initial interaction with the patients.

Although it did not reach significance, probably due to insufficient case numbers, a trend of increased initial treatment compliance after the COVID-19 pandemic could be clearly observed. Little has been addressed on this issue regarding the changes in patient behavior compared to the impact on the healthcare system. As treatment in head and neck cancer is likely to take place in a virtual instead of telemedicine settings, doctors and other healthcare providers should be aware that these patients tend to stay at the nearest accessible facility to start their treatments once the diagnosis of head and neck cancer has been made. Thus, the hardware and software resource quantity, quality, and availability for the multidisciplinary team, including surgeons, medical and radio-oncologists, psychiatrist, nutritionists, and social workers, should be re-examined and reinforced if the current resources are incapable of managing the expected increase in the volume of these patients after the COVID-19 pandemic.

There are several limitations to our study. First, the selection bias issue, as seen in the retrospective study design, means that the proportion of patients visiting our hospital might not be evenly distributed, which could affect the actual disease incidence rate. Second, the address information documented might be different from the patient's actual residence, and the address of the caregiver was not investigated in this study. Thus, the transportation barrier demonstrated in this study should only be regarded as a rough estimation.

\section{Conclusion}

The primary site, overall stage, patient living distance to the $<$ blinded for review $>$ territorial dominion, and the impact of COVID-19 might affect the compliance of curative-intent treatment in patients with newly 
diagnosed head and neck cancers. While the impact on outcome remains to be determined, physicians and healthcare providers might consider adding more resources and strategies to enhance patient information and early patient participation with regard to shared decision making and to further lower barriers to patient transportation in order to increase the compliance of initiating the treatment in patients with head and neck cancers.

1. Kaidar-Person, O., Z. Gil, and S. Billan, Precision medicine in head and neck cancer. Drug Resist Updat, 2018. 40 : p. 13-16.

2. Rahman, Q.B., et al., Global Burden of Head and Neck Cancer.Oral and maxillofacial surgery clinics of North America, 2020.32 (3): p. 367-375.

3. Lin, S.S., S.T. Massa, and M.A. Varvares, Improved overall survival and mortality in head and neck cancer with adjuvant concurrent chemoradiotherapy in national databases. Head Neck, 2016.38 (2): p. 208-15.

4. Latter, S., et al., Perceptions and practice of concordance in nurses' prescribing consultations: Findings from a national questionnaire survey and case studies of practice in England.International Journal of Nursing Studies, 2007. 44 (1): p. 9-18.

5. Fesinmeyer, M.D., et al., Effect of radiotherapy interruptions on survival in medicare enrollees with local and regional head-and-neck cancer. Int J Radiat Oncol Biol Phys, 2010.78 (3): p. 675-81.

6. Mohanti, B.K., et al., Analysis of 2167 head and neck cancer patients' management, treatment compliance and outcomes from a regional cancer centre, Delhi, India. J Laryngol Otol, 2007. 121 (1): p. 49-56.

7. Liao, D.Z., et al., Association of Delayed Time to Treatment Initiation With Overall Survival and Recurrence Among Patients With Head and Neck Squamous Cell Carcinoma in an Underserved Urban Population.JAMA otolaryngology- head \& neck surgery, 2019. 145 (11): p. 1001-1009.

8. Goel, A.N., et al., The impact of treatment package time on survival in surgically managed head and neck cancer in the United States. Oral Oncol, 2019. 88 : p. 39-48.

9. Graboyes, E.M., et al., Association of Treatment Delays With Survival for Patients With Head and Neck Cancer: A Systematic Review.JAMA otolaryngology- head \& neck surgery, 2019. 145 (2): p. 166-177.

10. Guttmann, D.M., et al., National disparities in treatment package time for resected locally advanced head and neck cancer and impact on overall survival. Head Neck, 2018. 40 (6): p. 1147-1155.

11. DiMatteo, M.R., et al., Patient adherence and medical treatment outcomes a meta-analysis. Medical care, 2002: p. 794-811.

12. Patel, U.A., et al., Poor radiotherapy compliance predicts persistent regional disease in advanced head/neck cancer. Laryngoscope, 2009. 119 (3): p. 528-33.

13. Chang, Y.L., et al., Factors impacting on discordance with treatment plan in head and neck cancer patients: a retrospective, population-based cohort study. Support Care Cancer, 2020.28 (2): p. 951-958.

14. Duvvi, S.K., et al., Two-week rule for suspected head and neck cancer. A study of compliance and effectiveness. J Eval Clin Pract, 2006. 12 (6): p. 591-4.

15. Chandarana, M.N. and P.S. Pai, Demography and treatment pattern of patients with head and neck carcinoma presenting to a tertiary care center in India: Need for urgent decentralization of cancer care. South Asian J Cancer, 2020. 9 (1): p. 38-42.

16. Chen, T.-J., L.-F. Chou, and S.-J. Hwang, Patterns of ambulatory care utilization in Taiwan. BMC health services research, 2006. 6 (1): p. 1-8.

17. Yang, Y.-T., et al., The relationship between accessibility of healthcare facilities and medical care utilization among the middle-aged and elderly population in Taiwan. International Journal for Quality in Health Care, 2015. 27 (3): p. 222-231. 
18. Bradley, E.H., et al., Variation in health outcomes: the role of spending on social services, public health, and health care, 2000-09. Health Affairs, 2016. 35 (5): p. 760-768.

19. Sturgis, E.M. and K.K. Ang, The epidemic of HPV-associated oropharyngeal cancer is here: is it time to change our treatment paradigms? Journal of the National Comprehensive Cancer Network, 2011.9 (6): p. 665-673.

20. Airoldi, M., et al., Functional and psychological evaluation after flap reconstruction plus radiotherapy in oral cancer. Head \& neck, 2011. 33 (4): p. 458-468.

21. Jehn, P., et al., Physical and psychological impairments associated with mucositis after oral cancer treatment and their impact on quality of life. Oncology research and treatment, 2019.42 (6): p. 342-349.

22. Ho, K.-Y., et al., Early recognition of nasopharyngeal cancer in adults with only otitis media with effusion. Journal of Otolaryngology-Head \& Neck Surgery, 2008. 37 (3).

23. Zullig, L.L., et al., Transportation - a vehicle or roadblock to cancer care for VA patients with colorectal cancer? Clinical colorectal cancer, 2012. 11 (1): p. 60-65.

24. Etminani-Ghasrodashti, R., C. Kan, and L. Mozaffarian,Investigating the Role of Transportation Barriers in Cancer Patients' Decision Making Regarding the Treatment Process.Transportation Research Record, 2021: p. 0361198121991497.

\section{Hosted file}

Table 1.docx available at https://authorea.com/users/422430/articles/528145-factorsaffecting-the-compliance-of-curative-intent-treatment-in-patients-with-head-and-neckcancer

\section{Hosted file}

Table 2.docx available at https://authorea.com/users/422430/articles/528145-factorsaffecting-the-compliance-of-curative-intent-treatment-in-patients-with-head-and-neckcancer

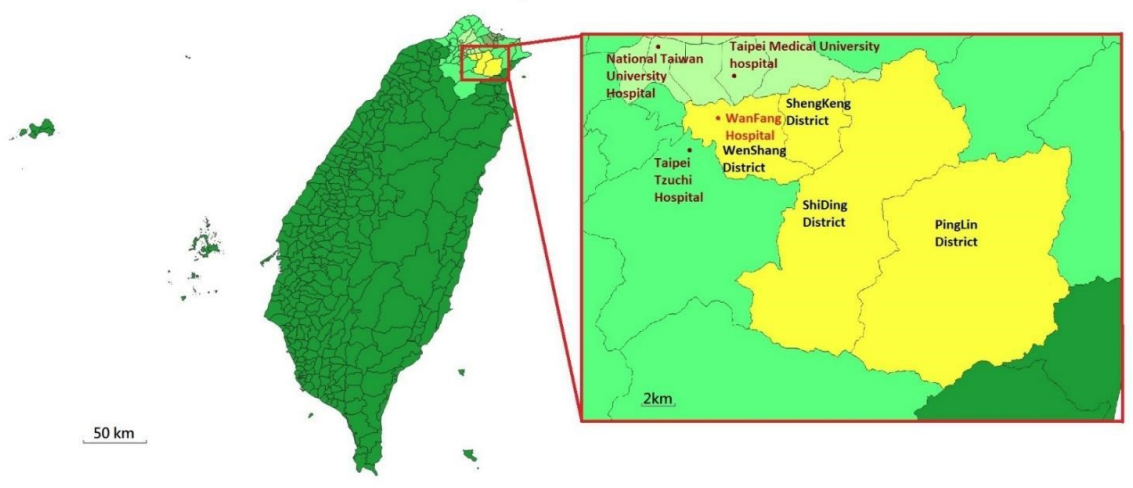

